\title{
Formación inicial docente y necesidades educativas especiales*
}

\author{
Initial teacher training and special educational needs \\ Formação inicial de professores e necessidades educativas especiais \\ Solange Tenorio \\ Departamento de Educación Diferencial. Universidad Metropolitana de Ciencias \\ de la Educación (UMCE), tel. 241-2515 241-2681. solange.tenorio@umce.cl
}

\begin{abstract}
Hace varios años, Chile viene haciendo diversos esfuerzos por mejorar la calidad y equidad del sistema educativo. Múltiples han sido las innovaciones y reformas para alcanzar mayores logros académicos en los estudiantes, estando éstas concentradas últimamente en mejorar la formación inicial docente e incentivar el acceso de mejores alumnos a las carreras de pedagogía. Los estudios respaldan la importancia de un buen profesor para obtener un aprendizaje de calidad y a su vez, ellos reconocen las nuevas y exigentes demandas laborales. Entre sus desafíos destaca el ser capaz de otorgar una respuesta educativa que sea apropiada a la diversidad del alumnado, en una sociedad que se declara a favor de la inclusión. En este contexto, el presente artículo da a conocer los principales hallazgos de una investigación realizada a estudiantes de cuarto año de distintas carreras de pedagogía de la UMCE acerca de su formación en temas relativos a la integración escolar, discapacidad, necesidades educativas especiales y su abordaje pedagógico.
\end{abstract}

Palabras Clave: formación inicial docente, discapacidad /necesidades educativas especiales integración/ inclusión escolar, calidad y equidad educativa.

\begin{abstract}
For several years Chile has been making diverse efforts to improve the quality and equity of the educative system. There have been multiple innovations and reforms to reach greater academic achievements in students, all these focused on improving the initial teaching training program and also on stimulating students with better results to enter pedagogical careers. The studies endorse the importance of a good professor in order to obtain quality learning, and as well, teachers recognize the new and arduous labor demands. Among the challenges teachers face, it stands out being able to grant appropriate educative answers to the diversity of pupils in the classroom in a society that declares itself in favor of the inclusion. In this context, this paper presents the main findings from an investigation made on fourth year students of different pedagogical specialties at UMCE, in relation to their own academic formation in subjects related to scholastic integration, disabilities, special aids, and their corresponding pedagogical approaches.
\end{abstract}

Palabras Claves: initial teaching training, scholastic integration, disabilities, special aids, educative quality and equity.

\section{RESUMO}

Há vários anos, o Chile vem realizando diversos esforços para melhorar a qualidade e a equidade do sistema educativo. Múltiplas têm sido as inovações e as reformas para alcançar maiores conquistas acadêmicas junto aos estudantes, concentrando-se na melhora da formação inicial docente e no incentivo do acesso dos melhores estudantes às carreiras pedagógicas. Os estudos reafirmam a importância de um bom professor para obter uma aprendizagem de qualidade e reconhecem, além disso, as novas e exigentes demandas profissionais. Entre os desafios, destaca-se

* Trabajo realizado con el financiamiento de la Universidad Metropolitana de Ciencias de la Educación, DIUMCE- APIS 08/10. 
o de ser capaz de outorgar uma resposta educativa que seja apropriada à diversidade de estudantes em uma sociedade que se declara a favor da inclusão. Neste contexto, o presente artigo apresenta os principais achados de uma pesquisa feita pelos estudantes do quarto ano de Pedagogia da UMCE, sobre sua formação em temas relativos à integração escolar, à deficiência, às necessidades educativas especiais e à sua abordagem pedagógica.

Palavras chave: formação inicial docente, deficiência/integração das necessidades educativas especiais/inclusão escolar, qualidade e equidade educativa.

\section{INTRODUCCIÓN}

Nuestro país, desde la década del noventa, ha iniciado una serie de reformas en el ámbito educativo, con la proclamada y decidida intención de mejorar la calidad y equidad del sistema educacional chileno. En estos veinte años se ha implementado una serie de medidas y programas para introducir mejoras en los procesos educativos que se traduzcan en el logro de mejores aprendizajes de los estudiantes.

Entre las medidas por las que se ha transitado destacan la reforma curricular, la puesta en marcha de la Jornada Escolar Completa, los programas MECE (de Mejoramiento a la Calidad y Equidad) a la educación básica y media, el apoyo al Perfeccionamiento y Evaluación Docente, el Sistema de Aseguramiento de la Calidad y Gestión Escolar (SACGE), la Ley de Subvención Escolar Preferencial para estudiantes vulnerables y la puesta en marcha de los sistemas de acreditación con la respectiva Comisión Nacional de Acreditación, entre otros. Una importante medida tomada desde el ejecutivo en el último gobierno de la Concertación fue la conformación del Consejo Asesor Presidencial y la firma del "Acuerdo para la Calidad de la Educación", el 13 de noviembre de 2007. "Este acuerdo indicaba que los nuevos principios prioritarios del sistema educacional chileno serán Calidad, Igualdad, Rendición de cuentas y Transparencia" (OCDE, 2009:29). En él se definió, entre otros puntos, la responsabilidad del Gobierno de proteger los derechos de los estudiantes a recibir una educación de calidad; se estableció la necesidad de contar con una superintendencia para proteger los derechos de los estudiantes para las escuelas, sus dueños y sus familias, y que se acordó formar una Agencia de Calidad para recoger la mejor información para el diagnóstico y solución de los problemas de la educación. Por otra parte, se instituyó la prohibición de selección de estudiantes hasta el $6^{\circ}$ año básico, situación identificada como la mayor causa de segregación social en el sistema escolar. (OCDE 2009). Cabe precisar que estas medidas fueron incorporadas en la nueva Ley General de Educación, promulgada el año 2009.

A pesar de la serie de innovaciones realizadas y los logros obtenidos, aún se está en deuda con los principios de calidad y equidad esperados, principalmente si se consideran los resultados obtenidos en pruebas internacionales.

En el transcurso de estos años varias han sido las agrupaciones, instituciones y expertos en materia educativa que han levantado propuestas e indicado algunas acciones necesarias de llevar adelante para lograr mejoras sustanciales en educación.

Entre estas organizaciones, el denominado Movimiento Ciudadano Educación 2020, agrupa sus propuestas en torno a 4 pilares fundamentales: profesores de excelencia, directores de nivel internacional, condiciones adecuadas para las aulas vulnerables, apoderados informados y participativos. "Estos cuatro pilares sólo pueden sustentarse en un sólido financiamiento público que resulta imprescindible para la materialización del conjunto de las propuestas" (Educación 2020, “en línea”). También precisan que para avanzar hacia un cuerpo docente de mejor formación, motivación y calidad el tema debe ser tratado 
desde varios ángulos, entre los que destacan: crear una Carrera Docente atractiva, trasformar la pedagogía en una profesión que conquiste alumnos de excelencia, mejorar la formación de aquellas instituciones que dictan Pedagogía, y "establecer un examen de habilitación obligatoria para ingresar a la Carrera Docente, al menos hasta que todas las instituciones pedagógicas del país tengan calidad homogénea y estándares curriculares mínimos". (Educación 2020, "en línea").

Por otra parte, en el informe emanado por el Panel de Expertos para una Educación de Calidad, denominado Propuestas para Fortalecer la Profesión Docente en el Sistema Escolar Chileno, se señala la necesidad de abordar tres áreas fundamentales: formación inicial docente, carrera docente y docentes directivos. El Panel considera que para el éxito de las propuestas, ellas deben ser abordadas integral y gradualmente, además de contar con la participación de todos los involucrados. (MINEDUC, 2010 a).

Cabe señalar que algunas de las propuestas merecen una discusión más a fondo, la que amerita ser abordada en otra ocasión, ya que son claramente cuestionables por tender a la segregación de un grupo importante de estudiantes. Podría ser entendida como una medida discriminatoria, para aquellos que distan de ser denominados "alumnos de excelencia", ese grupo que no alcanza los mejores puntajes en las prueba de ingreso a la universidad, pero sí pueden sentirse verdaderamente motivados por ser profesores. No se debe olvidar que entre los factores que explican un bajo puntaje PSU (Prueba Selección Universitaria) destacan las enormes desigualdades sociales y educacionales aún existentes en nuestro país, por lo que los resultados académicos o de rendimiento son una clara consecuencia de las falencias de nuestro sistema educativo. Sin embargo, acerca de lo que sí hay consenso, es en la necesidad de mejorar la formación inicial docente, particularmente si las carreras de pedagogía no reciben precisamente a aquellos de mayor excelencia académica. En este sentido, la tarea debiese concentrarse en hacer un mayor esfuerzo por entregar las herramientas necesarias para lograr un buen desempeño profesional de todos los que egresan de pedagogía. Es por ello que el factor formación docente debiese ser intervenido para lograr a corto y mediano plazo importantes progresos en la calidad del sistema educacional. Esto, fundamentado en investigaciones que evidencian el peso explicativo que tiene un buen profesor en el logro del rendimiento académico de sus alumnos.

Los estudios muestran que en los países desarrollados un factor determinante lo constituyen la familia y la comunidad (peso de $80 \%$ ). Sin embargo, en países en vías de desarrollo como el nuestro, los factores relativos a la escuela son de mayor importancia para explicar la varianza en el rendimiento de los alumnos. (Brunner y Elacqua, 2004).

La efectividad de la escuela aparece determinada, principalmente, por la efectividad del profesor en el aula, es decir, de la calidad de la docencia que éste imparte. También influyen variables como: las prácticas de enseñanza del docente en la sala de clase, el desarrollo profesional docente y los insumos (tamaño del curso, educación inicial y experiencia del profesor. (Brunner y Elacqua, 2004).

La importancia de factores relacionados con la escuela también ha sido demostrada en estudios de efectividad escolar en Chile (Bellei et al. 2004). Estos estudios recalcan la importancia del factor docente en el logro de aprendizajes, entendido como rendimiento académico.

Sin embargo, asimilar el término efectividad escolar a rendimiento académico, implica un reduccionismo difícil de aceptar. Se debe subrayar que una escuela es eficaz si además de obtener óptimos rendimientos escolares, principalmente buenos resultados en lenguaje 
y matemática, se preocupa de la formación en valores, del bienestar y satisfacción, y del desarrollo integral de los alumnos. También cabe agregar que eficacia y equidad son principios mutuamente necesarios, esto significa que una escuela, para ser eficaz, debe favorecer el desarrollo de todos y cada uno de sus alumnos (Murillo, 2003).

Para el logro de la calidad educativa necesariamente la escuela debe ser inclusiva y romper con las inequidades del sistema educacional, que se traducen en diversas barreras para el aprendizaje.

En este contexto, la educación no sólo debe tomar en consideración las necesidades de la mayoría, sino que ofrecer una respuesta educativa concreta para los alumnos que provienen de distintas etnias, culturas, de ambientes deprivados socioeconómicamente o marginados, y para aquellos niños y jóvenes que producto de alguna discapacidad no han podido participar de la escuela regular.

Lo anterior implica nuevos escenarios y espacios socioculturales-educativos, los que se transforman en requerimientos para la formación y profesionalidad docente, generan nuevas condiciones de inserción y acción profesional. Por ello, los docentes deben estar preparados para responder a las nuevas demandas sociales.

\section{ANTECEDENTES SOBRE ACUERDOS Y NORMATIVAS}

En esta dirección, el MINEDUC ha propiciado también importantes avances en el área de la educación especial, impulsados por los acuerdos en el ámbito de los derechos humanos, sociales, económicos y culturales de las personas, lo que ha implicado una nueva política y decretos que permiten mejoras sustanciales en términos de acceso y participación en el sistema escolar de estudiantes que presentan alguna discapacidad.

Un ejemplo claro de lo anterior, fue la promulgación de la Ley de Integración Social de Personas con Discapacidad No 19.284 del año 1994, la que no sólo abría oportunidades de acceder a una mejor educación, sino también a una mejor salud, recreación y trabajo, mejorando así la calidad de vida de niños y jóvenes. Ese año, el Ministerio de Educación, representantes de organizaciones y entidades sostenedoras de establecimientos educacionales suscribieron el "Acta de Compromiso por la Integración de Niños, Niñas y Jóvenes con N.E.E.” (Tenorio, 2005).

Para cumplir con lo dispuesto en la Ley № 19.284 en lo referido al ámbito educativo, se promulgó el año 1998 el Decreto No 01 "Integración escolar de alumnos y alumnas con necesidades educativas espaciales", y en el año 1999 el Decreto No 374 . En ellos se establecen alternativas educativas para brindar opciones que permiten y facilitan el acceso, permanencia y progreso en el sistema escolar común, indicando la necesidad de efectuar adecuaciones curriculares. Asimismo, posibilita a través de la creación de proyectos de integración escolar (PIE) obtener recursos para implementar esta iniciativa.

Cabe señalar que el MINEDUC ha estimulado a las escuelas regulares a integrar en sus aulas a estudiantes con discapacidad, intentando responder por una parte a los cambios en la forma de concebir el aprendizaje y la discapacidad, y por otra, a la necesidad del Estado por aumentar la cobertura de alumnos con N.E.E. en las escuelas regulares, debido a la presión de los organismos internacionales que condicionan fondos al cumplimiento de objetivos de inclusión, entre otros. (Tenorio, 2005). 
La década del noventa se caracterizó por el diseño de políticas educativas y estrategias que promovieron la generación de condiciones que facilitaron la integración escolar. Se adoptaron medidas legales que por una parte posicionaron la temática configurando una realidad, y por otra, se intentó regular o normar a través de orientaciones generales los procedimientos y modalidades, para otorgar una alternativa real de acceso, permanencia y progreso en el sistema escolar común.

Con la Política Nacional de Educación Especial 2005 se inicia una nueva etapa en esta área, estableciéndose los lineamientos para que alumnos/as con necesidades educativas especiales (N.E.E.) hagan efectivo el derecho a la educación de calidad, a la igualdad de oportunidades, a la participación y a la no discriminación. Se subraya el firme propósito de avanzar en comunidades más inclusivas y respetuosas de la diversidad (MINEDUC, 2010 b). Uno de los puntos centrales de dicha política, ha sido impulsar con gran decisión la integración escolar de alumnos con alguna discapacidad y trastornos específicos del lenguaje al sistema escolar común. Para materializar lo anterior, ha utilizado la capacidad institucional pública: escuelas y liceos municipalizados y particulares subvencionados principalmente.

La integración escolar se ha fundamentado principalmente en una opción ideológicocultural en favor de las minorías y en la exigencia social y económica de otorgar igualdad de oportunidades a personas, que al estar en un sistema especializado de educación, terminan excluidas socialmente. (Marchesi et al. 2001).

La Ley General de Educación (LGE), por su parte, también ha realizado un significativo aporte en relación a los alumnos que presentan N.E.E., relevando un trato preferencial con ellos, creando diversas condiciones para asegurar su progreso educativo en igualdad de condiciones con sus pares. Para ello, ha subrayado la necesidad de avanzar en el ámbito curricular en al menos tres dimensiones: modernización del currículo, flexibilidad y cobertura de poblaciones específicas, y evaluación y certificación de aprendizajes y competencias. (MINEDUC, 2010 b).

Cabe señalar que la antigua Ley Orgánica Constitucional de Enseñanza no hacía referencia a la educación especial, a diferencia de la LGE que precisa que ésta es una modalidad del Sistema Educativo, que desarrolla su acción educativa en los distintos niveles del sistema escolar, tanto de los establecimientos de educación común como especial. (MINEDUC, 2010 b). Este hecho denota una nueva mirada y preocupación del nivel central en la búsqueda de mejorar los aprendizajes de todos los alumnos.

Desde las primeras "tímidas" experiencias de integración escolar, hasta el día de hoy, las cifras reflejan el aumento progresivo en la cantidad de alumnos integrados a las escuelas y liceos de nuestro país. Durante los años, la cobertura en la integración escolar de niños, niñas y jóvenes que presentan N.E.E., ha aumentado extraordinariamente. El número de matrículas de establecimientos con PIE en el año 2005 era de 41.023 y el 2009 fue de 68.117. Dicho crecimiento de matrícula se explica también por el aumento en la subvención de la educación especial. Los datos muestran que el gasto total en PIE en el 2005 en escuelas municipalizadas era de \$1.698.163.004, mientras que el 2009 ascendió a un total de \$34.057.168.313; en el sector particular subvencionado, el 2005 fue de \$664.010.256 y en el 2009 llegó a \$ 15.236.998.059. (MINEDUC, 2010 b).

A pesar de los importantes cambios y avances, la propuesta de integración escolar en Chile ha transcurrido gradualmente, y ha implicado una reflexión y un proceso de aprendizaje de experiencias, tanto nacionales como internacionales. Su implementación 
ha demandado importantes cambios en la escuela regular y la educación especial, los que no han estado ausentes de críticas, desinformación y dificultades para comprender las políticas planteadas. Se han suscitado ciertas tensiones entre los diversos actores del sistema escolar, originándose distintas concepciones de cómo entender e implementar esta innovación.

El fenómeno de la integración ha generado cierta controversia, en especial a la hora de discutir acerca de los beneficios concretos que pudiese acarrear a los niños y jóvenes que son educados dentro de esta modalidad. La educación en escuelas especiales es vista, por una parte, como una manera de cautelar la atención a las N.E.E. de un grupo de alumnos, pero por otra, es concebida como una manera de perpetuar la segregación escolar y también social de estos alumnos. Por tanto, la integración escolar como fenómeno educativo no es estimada de igual manera por los distintos actores en torno a los beneficios que ella podría acarrear a los niños con N.E.E. y al resto de los estudiantes (Tenorio, 2009).

Entre los propios docentes de las escuelas existen visiones diferentes respecto al tema, las que están influidas por una serie de factores entre los que destacan las "características de la organización escolar y su gestión, la cultura escolar que impera en el centro escolar y la formación inicial y el perfeccionamiento docente recibido, principalmente en torno a la diversidad y concepciones del aprendizaje, que se traducen en una determinada práctica pedagógica". (Tenorio 2005: 828).

La década del 2000 se ha destacado por la realización de una serie de estudios e investigaciones tendientes a tomar el pulso de la situación educativa de los estudiantes con N.E.E. dentro del sistema regular y evaluar el resultado de la integración a nivel nacional, intentando identificar aquellos facilitadores y obstaculizadores en su implementación. Estos estudios han permitido el diseño de algunas modificaciones y/o precisiones, traducidos en instructivos y decretos para mejorar aquellos puntos detectados como críticos o débiles, creando condiciones más acordes a la realidad de las escuelas y sus alumnos con N.E.E.

Un producto concreto de ello fue el instructivo $\mathrm{N}^{\circ} 191$ del 2006, que tiene por objetivo clarificar algunos aspectos del Decreto 01/98, normas de procedimientos específicos para la presentación, implementación y evaluación de los programas de integración escolar, de modo de garantizar la calidad de los procesos educativos de los alumnos integrados (MINEDUC, 2006). Ese mismo año se crea el Decreto $N^{\circ} 1398$, que otorga Licencia de Educación Básica a estudiantes que han cursado sus estudios con programas de estudio de la educación especial. (MINEDUC, 2010 b).

El año 2007 se promulga la Ley $\mathrm{N}^{\circ} 20.201$ que modifica el DFL 2 de subvenciones (de 1998), que incrementa los recursos financieros de la educación especial (subvención) a los estudiantes con N.E.E. más severas y complejas, ampliándose además a los estudiantes con déficit atencional y con dificultades de aprendizaje. El año 2009 a través del Decreto $\mathrm{N}^{\circ} 170$ se fijan las normas para determinar a los alumnos con N.E.E. que serán beneficiarios de las subvenciones establecidas en la Ley antes mencionada (MINEDUC, 2009). Esta medida constituye un nuevo incentivo para que las escuelas se involucren en procesos de integración, se mejoren las condiciones y otorguen mayores oportunidades para los estudiantes con N.E.E.

Producto de nuevas reuniones y acuerdos, a principios del 2010 se aprueba la Ley $\mathrm{N}^{\mathrm{o}} 20.422$ que establece normas sobre igualdad de oportunidades e inclusión social de 
las personas con discapacidad y que viene a reemplazar a la Ley $\mathrm{N}^{\circ} 19.284$. Actualmente se trabaja en los respectivos decretos para implementar sus propuestas.

Las normativas promulgadas en esta última década han significado un aporte fundamental para mejorar la calidad y equidad en la vida de personas que presentan discapacidad, siendo los reglamentos y decretos del ámbito educativo, acompañados por programas específicos de apoyo tanto a la docencia y la evaluación de las N.E.E., los que han sido desarrollados por diversas instituciones de educación superior de nuestro país. Sin embargo, es necesario seguir avanzando en mejorar la calidad y logros de aprendizaje de los estudiantes con N.E.E. y para ello resulta fundamental recoger la información obtenida de los estudios efectuados y tomar las medidas correspondientes.

\section{EVIDENCIAS QUE APORTAN LOS ESTUDIOS}

En nuestro país, los resultados de la investigación realizada por la UMCE durante los años 2006 y 2007 coinciden con la efectuada por CEAS el año 2004, en cuanto a que aún prima un modelo médico observado no sólo en educadores diferenciales, sino también en profesores de aulas regulares. En el primer estudio mencionado, se precisa que los profesores de aulas comunes consideran que son los profesores de apoyo los mayores responsables de la enseñanza de los/as alumnos/as con N.E.E. Se indica la urgencia de reorientar la formación inicial docente y optimizar la línea de formación continua de los profesionales involucrados en procesos de integración, considerando los nuevos enfoques teóricos respecto de la discapacidad. Se hace mención a la necesidad de desarrollar estrategias renovadas que consideren el marco curricular de la educación regular para asegurar el progreso educativo los/las estudiantes con N.E.E. (MINEDUCUMCE 2007).

En el estudio realizado por Tenorio sobre representaciones sociales de profesores de enseñanza básica acerca de la integración escolar, se menciona que gran parte de los docentes consultados tienen una representación social de la integración escolar más bien negativa, ya que "destacan las dificultades que han presentado en su desempeño profesional y en el progreso académico de los alumnos integrados; asimismo, hacen referencia a una serie de barreras en la implementación de su política" (2007:338). Si bien se destaca que existe la disposición y necesidad por parte de los profesores de introducir transformaciones e innovaciones en las prácticas pedagógicas, dicha intención se ve limitada por tener fuertemente incorporada una concepción tradicional (modelo médico) acerca de la discapacidad y del rol de la educación especial y regular. Este mismo estudio concluye que aquellos docentes que habían tenido experiencias previas en integración escolar, o bien habían tenido interacciones en otros ámbitos con personas con algún tipo de discapacidad, presentaban una representación social claramente más positiva en comparación con aquellos profesores que no habían pasado por tales experiencias.

Otro estudio efectuado en Asturias, España, relacionado con las actitudes de los docentes ante las necesidades educativas especiales, destaca que más del $20 \%$ de los maestros desconoce lo "que realmente significa que un alumno presente necesidades educativas especiales, y sin embargo, estén escolarizados en sus aulas" (Álvarez et al. 2005: 605). Se indica que esta situación revela la falta de información y probablemente de formación en el tema. Se recalca el hecho de que existe en los docentes la disposición al trabajo de 
alumnos con N.E.E., pero no la respectiva preparación, la que se refleja en gran medida por actitudes de compasión ante estos estudiantes y por considerar que entorpecen el funcionamiento de la clase. Coincidentemente con esta investigación, una realizada también en España (Provincia de Albacete), concluye que existe cierta resistencia a la integración escolar y entre los factores que influyen se nombran la falta de información, carencia de apoyo especializado y el deficiente nivel de formación. (Montañés et al. 1990).

Ya en el año 1985, García y Alonso concluían que se observaban actitudes más positivas hacia la integración escolar en aquellos maestros que se consideraban más informados en el tema.

El estudio realizado por Sola concluyó que la formación coherente y bien planificada tanto inicial como permanente de los profesores, es uno de aquellos factores que pueden propiciar actitudes positivas y/o negativas de los docentes ante la diversidad y su inclusión. Asimismo, la investigación evidencia que la falta de formación adecuada podría ocasionar desinterés o rechazo hacia la integración de alumnos con necesidades educativas especiales a la escuela común. (Sánchez y Torres 1997).

Varios son los autores (Stainback y Stainback, 1999 y Arnaiz 2003), que afirman que para el logro de la inclusión educativa, se requiere de profesores formados para dar respuestas educativas a la heterogeneidad de sus alumnos.

Burke y Sutherland, 2004, destacan la relación positiva entre la experiencia y el conocimiento de los docentes respecto de los alumnos con discapacidad y actitudes hacia la inclusión.

Los diversos estudios reflejan la importancia de las actitudes de los docentes hacia la integración de los alumnos con N.E.E., la que está mediada por la formación profesional, entendida como conocimiento de la temática y capacidad de dar una respuesta educativa a los alumnos que lo requieren. Ellos se constituyen en un factor facilitador o bien en una barrera para el logro de una real integración. Esto cobra especial sentido, considerando que son las escuelas públicas y de bajos recursos aquellas en las que se ha focalizado la implementación de la integración escolar, recibiendo a estudiantes que por su propia discapacidad presentan un factor de mayor vulnerabilidad asociado.

Por su parte, la OCDE reconoce el aumento progresivo en nuevas exigencias a los profesores en su trabajo cotidiano y en las expectativas sobre lo que implica ser un buen docente. La noción de docente efectivo está mediada por habilidades para facilitar la inclusión educativa, el manejo de las TICs, por la capacidad de evaluar a sus estudiantes e investigar para mejorar su práctica docente. En el contexto del estudio TALIS (2007-2008), los mismos docentes afirman que les hubiera gustado tener más actividades de desarrollo profesional relacionadas con la enseñanza de estudiantes con necesidades especiales (32\%, correspondiente porcentaje más alto de preferencias). Este organismo reconoce "la existencia de una demanda insatisfecha en un número importante de países, que se refiere a áreas como la educación inclusiva...”. (OCDE, 2009: 5). También admite lo difícil que resulta que los profesores estén preparados para todos estos desafíos y para los nuevos que vayan surgiendo, si no existe una oferta de desarrollo profesional docente actualizada y flexible.

Tal como señala el Panel de Expertos para una Educación de Calidad, "nuestro país ha experimentado una expansión de la oferta de carreras de pedagogía, sin que se haya puesto atención en la calidad de dichos programas ni tampoco en el reclutamiento de jóvenes más preparados para tan decisivos estudios". (MINEDUC, 2010 b: 6). Sin lugar a dudas, que el primer objetivo relacionado con la calidad de los programas de 
pedagogía debe ser abordado desde múltiples aristas, considerando dar respuesta a las actuales exigencias de la sociedad, lo que implica formar docentes con competencias para el trabajo con estudiantes con N.E.E.

La Política de Educación Especial explicita entre sus líneas estratégicas y acciones a impulsar entre los años 2006 y 2010 la necesidad de mejorar la formación inicial y en servicio de los docentes y profesionales de la educación especial y regular, para ello se propuso favorecer "un trabajo conjunto con las universidades e institutos profesionales que imparten las carreras pedagógicas para que éstas incluyan en sus mallas curriculares conocimientos y estrategias para educar en la diversidad y atender las necesidades educativas especiales" (MINEDUC, 2005: 58). Tomando en consideración que este objetivo ya debiese estar siendo desarrollado, resulta necesario hacer un diagnóstico a la realidad y ver en qué medida se está cumpliendo.

\section{LA INVESTIGACIÓN}

En este contexto y considerando la problemática planteada, los estudiantes egresados de la carrera Licenciatura en Educación y Pedagogía en Educación Diferencial, especialidad Problemas de Aprendizaje: Gabriela González, Javier Herrera y Eva Jara, llevaron a cabo una investigación en la UMCE guiada por la autora de este artículo. Este estudio tuvo la finalidad de describir la formación recibida por estudiantes de distintas carreras de pedagogía, en materia de integración escolar y estrategias didácticas para el abordaje de las N.E.E. La muestra estuvo constituida por 80 estudiantes, 10 de cada una de las siguientes carreras: Licenciatura en Educación y Pedagogía en Arte, Educación Física, Biología, Matemática, Castellano, Historia y Geografía, Educación Básica y Párvulo.

Éste correspondió a un estudio mixto, cuali- cuantitativo, en el que se emplearon como procedimientos para recoger información: un cuestionario, entrevistas semi estructuradas y selección documental (mallas curriculares). Se efectuó un análisis de contenido de la información cualitativa obtenida y para lo cuantitativo, se recurrió a estadística descriptiva. Dicho análisis aportó información en distintos niveles: por carrera, facultad y finalmente considerando toda la muestra de los estudiantes de las carreras de la UMCE que formaron parte del estudio. A continuación se dan a conocer algunos resultados obtenidos, principalmente del último nivel indicado.

\subsection{ALGUNOS HALLAZGOS}

A continuación se presentan algunos resultados obtenidos y sintetizados en las siguientes categorías:

- Visión acerca de la integración escolar, la discapacidad o N.E.E.

Un porcentaje mayoritario de estudiantes (65\% del grupo de estudio) piensa que los docentes de aula regular tienen la responsabilidad educativa de trabajar con estudiantes con discapacidad o N.E.E., y que ésta no sólo recae en los educadores diferenciales. Sin embargo, el $84 \%$ considera que profesores de aula común no están preparados para la integración escolar y el $93 \%$ de la muestra opina que dichos docentes debieran tener las herramientas necesarias para responder a las N.E.E. de sus alumnos, ya que éstos debiesen 
recibir estrategias pedagógicas distintas que el resto de sus compañeros. La mayoría de los participantes cree que existen discapacidades más fáciles o difíciles de trabajar en el aula y afirman que la presencia de alumnos con N.E.E. no afecta de manera negativa el rendimiento de un curso.

En los estudiantes no existe una clara tendencia a favor de la integración, si bien un alto porcentaje (un $43 \%$ del total de la muestra) se encuentra en total desacuerdo con la afirmación referida a que los estudiantes con discapacidad debieran estar en escuelas especiales, sólo un $16 \%$ se manifiesta por el acuerdo y el $41 \%$ de la muestra no tiene una opinión clara frente al tema. Esta información es coherente con lo que reflejan los datos ante la afirmación "como profesor, preferiría no tener estudiantes con N.E.E. en la clase", ya que un $59 \%$ se manifiesta en desacuerdo frente a la afirmación, mientras que un $30 \%$ indica no estar de acuerdo ni en desacuerdo y un $11 \%$ señala estar de acuerdo con la aseveración.

- Formación inicial docente en relación a Integración Escolar y abordaje de las Necesidades Educativas Especiales

Del total de estudiantes de la muestra, el $44 \%$ afirma haber abordado durante su formación temáticas relacionadas con la Integración/Discapacidad y acerca del trabajo con estudiantes que presentan N.E.E. Indican haberlo hecho principalmente en una actividad curricular planificada en clases, otros en trabajos de investigación y también en menor número en conversaciones con docentes interesados en el tema. El otro $56 \%$ de los estudiantes afirma no recordar haberlas tratado. Sólo el $25 \%$ afirma haber participado en prácticas con estudiantes con N.E.E. integrados.

Finalmente, ante la afirmación "las herramientas para trabajar con estudiantes con N.E.E. las encontré fuera de la carrera", el $39 \%$ de la muestra señala estar de acuerdo con esta afirmación, el $38 \%$ indica no estar de acuerdo ni en desacuerdo, mientras que el $24 \%$ de las estudiantes declara estar en desacuerdo con la afirmación.

La mayoría de los estudiantes señalan que durante su formación sólo los han preparado para trabajar con estudiantes sin N.E.E. en el aula. El 59\% marca estar de acuerdo con la afirmación: "en tu carrera sólo te han preparado para trabajar con estudiantes sin N.E.E.”, un $35 \%$ se muestra de forma neutral ante la afirmación y un $6 \%$ se manifiesta de acuerdo con ella.

Cabe señalar que de la totalidad de las carreras participantes en la investigación, son los estudiantes de la carrera de Educación Básica quienes reconocen haber sido formados en torno al tema, ya que poseen una cátedra en VIII semestre referida a los Problemas de Aprendizaje. Gracias a esta actividad curricular, tuvieron la oportunidad de abordar conceptos, reconocer las N.E.E. y analizar documentos de manera crítica y reflexiva. En la carrera de Educación Parvularia, las estudiantes también reconocen la existencia de formación en estos temas, trabajados en variadas cátedras a través de análisis de documentos, conceptos, trabajos de investigación y presentaciones, entre otras. No obstante, pese a reconocer lo anterior, existe un grado de disconformidad, por cuanto consideran que la preparación ha sido sólo a nivel teórico. Otros manifiestan que una sola cátedra durante los años de estudio es insuficiente para dominar el tema y estar preparados para enfrentar en la práctica misma la atención a la diversidad: "se trata el tema muy a la ligera, falta mayor profundización”. 
El 95\% de los estudiantes valoraría la inclusión de actividades curriculares relacionadas con la temática en la malla curricular, un $1 \%$ se muestra en desacuerdo de incluir estos temas y un $4 \%$ se muestra neutral.

Ante la pregunta "Si tuvieras que incluir temáticas/actividades curriculares relacionados con la integración y abordaje de las N.E.E. durante tú formación, las incluirías en...”, el $41 \%$ prefiere que se aborden en la línea de licenciatura, un 34\% optan por incorporarlas en el área o línea de la carrera (especialidad), y un 25\% se inclina por tenerlas como electivos u optativos.

- $\quad$ Competencias para el trabajo con estudiantes con N.E.E.

En lo que respecta a aquellas competencias que consideran debiesen desarrollarse durante la formación docente para enfrentar el proceso educativo de estudiantes con N.E.E., el orden de preferencias de las competencias dado por los estudiantes es el siguiente:

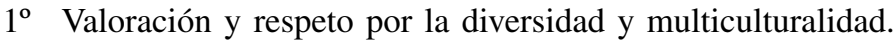

$2^{\circ}$ Capacidad para identificar, plantear y resolver problemas.

$3^{\circ}$ Capacidad de aplicar los conocimientos en la práctica.

$4^{\circ}$ Capacidad de aprender y actualizarse permanentemente.

$5^{\circ}$ Capacidad creativa.

La información de orden cuantitativa recientemente dada a conocer es totalmente coherente con aquella recogida a través de preguntas abiertas en las que los estudiantes expresan debilidades y fortalezas de su formación en materia de integración escolar y abordaje de las N.E.E.

- Debilidades de la formación inicial docente y propuestas para su mejora

En cuanto a debilidades, la mayoría de los estudiantes reconoce como una debilidad en su formación la falta de preparación en los temas relacionados con la diversidad en el aula, Integración Escolar, estrategias pedagógicas para el trabajo de estudiantes con N.E.E. Fundamentan que esta situación podría deberse al enfoque de formación de las carreras, en donde se prioriza el "saber disciplinario" por sobre el "saber pedagógico" más general, dejando en claro que si en algún momento se hizo referencia al tema, esto fue de manera superficial y sin mayor profundización. "Poco y nada es lo que se abordan estas temáticas en el aula”. Pese a lo anterior, los estudiantes reconocen la importancia de tratar temáticas relacionadas con las N.E.E. durante su formación, aludiendo a la necesidad imperiosa de ser preparados no sólo en aspectos meramente teóricos, sino que también en la enseñanza de estrategias y metodologías que permitan en la práctica pedagógica responder a las necesidades de aquellos estudiantes integrados y/o con necesidades educativas especiales. Algunos manifiestan que esta temática debiese ser parte de la formación transversal de todas las carreras de pedagogía, independientemente de la especialidad o la disciplina estudiada, fundamentando que todo profesor debiese estar preparado para atender y trabajar con la diversidad de sus estudiantes: "Que no se nos ha enseñado ni hablado de esta temática, es un tema que nos compete a todos los profesores y que es necesario que dominemos no sólo el tema, sino que estrategias metodológicas también". 


\section{- Fortalezas de la formación inicial docente}

En lo que se refiere a fortalezas, en primera mayoría, un considerable número de estudiantes no reconoce mayores fortalezas en su formación docente en cuanto a estas temáticas, afirmando en algunos casos que estos temas nunca fueron abordados y si lo fueron, no se les daba mayor importancia o profundización. Una cantidad menor reconoce como fortaleza el desarrollo de algunas competencias fomentadas dentro de sus carreras, que siendo adaptadas podrían ser aplicadas al trabajo con estudiantes integrados y/o con N.E.E. Estas competencias son la capacidad creativa, indagativa, reflexiva, entre otras. También se agrega como fortaleza la enseñanza de estrategias y metodologías, y pese a que no estaban enfocadas a su aplicación con estudiantes con N.E.E., revelan cierta seguridad de poder adecuarlas al contexto: "Que pese a que es un tema que no se ha tocado, creo que la capacidad reflexiva e indagativa y resolver problemas, son competencias que hemos desarrollado en nuestra carrera y que nos pueden servir en esta temática".

Un grupo menor realiza una valorización positiva de la formación con respecto a la temática. Señalan que ella les ha permitido acercarse a contenidos, conceptos y sensibilizarse frente al tema en cuestión, considerando un aporte la presencia de una cátedra en su malla curricular. En otra carrera, sus estudiantes se refieren al abordaje de las temáticas en instancias como conversaciones, trabajos de investigaciones, lectura crítica de documentos, entre otras. “...nos entregan teoría bastante consistente, análisis crítico de los documentos".

Algunos reconocen que durante su formación se hace necesario poder tener más preparación al respecto; no obstante, consideran como fortaleza aquellas experiencias adquiridas en otras instancias o situaciones ajenas al proceso educativo formal. Otros reconocen como fortaleza la iniciativa e interés propio por conocer y formarse en este aspecto: "Tuve la oportunidad de realizar una práctica en la Teletón, pero fue solo por iniciativa del profesor y no porque sea de la malla".

Por otra parte, la investigación incluyó entrevistas tendientes a conocer la conceptualización que poseen de algunos términos y de esta forma comprender el paradigma que subyace a ellos.

\section{- $\quad$ Conocimiento de conceptos básicos}

En relación al término Necesidades Educativas Especiales, es posible encontrar una serie de dimensiones en las que centra el concepto. La mayoría de los estudiantes manifiesta que este concepto es relativo a las necesidades que presentan los profesores para responder a las carencias de sus alumnos. También se asocia este concepto con las necesidades que presentan los estudiantes con problemas para tener una educación de calidad. Algunas afirmaciones que ejemplifican esta categoría son: "Son las necesidades que tienen ciertos alumnos y también profesores para abarcar las carencias educativas de alumnos con problemas".

La segunda mayoría de respuestas son aquéllas que asocian el concepto de las N.E.E. con los problemas de aprendizaje. Se precisa que son necesidades específicas que presentan algunas personas debido a algún problema en particular: "Son las necesidades que tienen ciertos alumnos que poseen alguna dificultad en su aprendizaje y que por esta razón debe adecuarse el proceso de enseñanza-aprendizaje”. También relaciona las N.E.E con los estudiantes que poseen problemas de integración. La mayor parte de las respuestas son vinculadas con las personas que posean algún grado de discapacidad, ya 
sea física o cognitiva: "Creo que la sigla se orienta hacia los alumnos que carecen de algún aspecto físico y que requieren ayuda”.

Una menor cantidad de respuestas relacionan el concepto de las N.E.E. con las distintas metodologías y materiales que deben utilizar los docentes para responder a los requerimientos que poseen algunos estudiantes, así como también con la necesidad de introducir cambios en la infraestructura de los establecimientos.

Si bien existen énfasis diferentes en las respuestas, es posible indicar que dos grupos se aproximan a un enfoque teórico más actual, evitando centrarse exclusivamente en déficit o dificultades de los alumnos, dando la debida responsabilidad al docente y a la escuela al otorgar la respuesta educativa apropiada.

En cuanto a Integración Escolar, la mayoría de los estudiantes comprende el concepto como la incorporación de estudiantes con N.E.E. o con discapacidad a la escuela regular. "Incorporación de estudiantes con diversas discapacidades que antes eran tratados en escuelas especiales, a los establecimientos educacionales formales".

Con un menor número de respuestas, relacionan el concepto de la integración escolar con "no discriminar", tomando en consideración para contestar uno de los fines de la Integración Escolar. "Medida de poder integrar a los párvulos al sistema educativo y no discriminar por algún tipo de discapacidad que presente".

Finalmente, el menor porcentaje de respuestas asocia el concepto de integración escolar con el concepto más amplio de inclusión escolar. "Corresponde a la posibilidad de abrir los establecimientos educacionales a todas las personas respetando y valorando toda la diversidad humana".

Cabe señalar que gran parte de las respuestas reflejan esta posibilidad que da la escuela común, al abrir un espacio a otros que antes no pertenecían a ese lugar, pero no se hace referencia al beneficio mutuo de participación y aprendizaje conjunto de niños con y sin N.E.E.

En lo que al término discapacidad se refiere, la gran parte de los entrevistados lo define como una limitación física o cognitiva propia del ser humano y que le impide desarrollarse en su totalidad o plenitud: "Problemas motrices, físicos o mentales que posee alguien y dificulta el desarrollo de ciertos procesos". "Son personas que están en cierta desventaja con personas que no las poseen. Como tener problemas visuales, pérdida de audición, etc.”.

Finalmente una minoría de estudiantes hace referencia a ella como una situación que puede sobrellevarse y no limitar el desarrollo personal. "Situación que limita al sujeto. Sin embargo, puede sobrellevarse y realizar una vida normal".

En esta última conceptualización, está la idea de otorgar las herramientas necesarias que posibiliten al sujeto el desarrollo de una vida común, la que se aproxima a un paradigma más actual en torno al término. En este enfoque se subraya la responsabilidad de toda la sociedad de crear las condiciones óptimas para lograr el desarrollo integral de las personas.

El concepto de inclusión es mayoritariamente considerado como un sinónimo de "integración escolar", es decir, como el ingreso de niños con algún tipo de discapacidad o que presentan alguna N.E.E. ante el sistema educativo regular. Algunas afirmaciones que reafirman lo anterior son: "Incluir a personas discapacitadas a la sala de clases".

Otro grupo de respuestas vinculan el concepto de inclusión con la no discriminación de las personas, teniendo en cuenta las características que puedan diferenciarlos. "No 
considerar ni practicar la discriminación de ningún tipo". Algunos se refieren a él como un proceso en donde se integra a las personas, sin importar la clase social, religión, sexo y características entre otros factores que puedan ser relevantes. "Aceptación de todos los alumnos, sin importar su raza, religión, capacidades, etc”.

Finalmente, un grupo minoritario relaciona el concepto de "inclusión" con la participación activa de las personas dentro de un sistema. De esta categoría se desprenden afirmaciones tales como: "Es incluir a jóvenes para que participen y se sientan valorados".

Cabe agregar que los participantes del estudio que vinculan el concepto de inclusión con términos como no discriminación, participación, valoración, se aproximan a referentes teóricos actuales, y al sentido que se espera que éste tenga para la sociedad en general, y no sea acotado exclusivamente al ámbito de la integración escolar.

El término adecuaciones curriculares es dominado por la mayoría de los estudiantes, sin embargo, realizan algunas distinciones centrando el énfasis en distintos aspectos del concepto. Una de las dimensiones que se considera en las respuestas dice relación con los beneficiarios de tales adecuaciones.

El mayor número de actores estudiados comprende el concepto de adecuaciones curriculares como los cambios que deben realizarse en el curriculum, con motivo de poder integrar a niños con N.E.E. dentro del sistema educacional regular. Algunas afirmaciones que se desprenden de ésta categoría son: "Creo que puede tener relación con modificaciones en el currículo, que permitan una mayor integración de estudiantes con discapacidad".

Otros se refieren a él como la adaptación y mejoras que se producen en el currículum oficial en beneficio de una educación de calidad para todos los estudiantes. Algunos hacen referencia a la flexibilidad que debe tener el currículo para ser capaz de adaptarse a distintas situaciones y responder al contexto. "Modificación del currículo a fin de adaptarlo al contexto del establecimiento y de los estudiantes, que permite la aplicación en el aula".

\subsection{ALGUNAS CONCLUSIONES}

A pesar de lo declarado desde el nivel central, y particularmente considerando las acciones propuestas por la Política de Educación Especial en relación a favorecer un trabajo colegiado con universidades para que las carreras pedagógicas incorporen en sus mallas curriculares conocimientos y estrategias para educar en la diversidad, los resultados de esta investigación indican que aún se está en deuda con dichas propuestas e iniciativas.

Los temas acerca de las N.E.E. y su abordaje pedagógico aún no forman parte de una política institucional que los considere esencial en la formación del profesor. Es posible visualizar que sólo algunas carreras han innovado en este sentido. Las mallas curriculares de la mayoría de las carreras estudiadas no tienen incorporadas actividades orientadas al trabajo pedagógico pensando en la diversidad del alumnado. Solo en carreras de la Facultad de Filosofía y Educación se observa una mayor aproximación al tema. La carrera de Educación Básica posee una asignatura en su malla curricular con dicha orientación, y en la carrera de Párvulos se aborda el tema en distintas actividades como investigaciones, análisis de documentos y conversaciones. A pesar de ello, la gran mayoría de los estudiantes de la UMCE reconoce la importancia de trabajar las temáticas 
y se manifiestan a favor de incorporarlas dentro de su formación. Esta posición, así como las conceptualizaciones que manejan, permite afirmar que en ellos existe conciencia de la relevancia del desarrollo de competencias en esta área para un óptimo desempeño profesional. También se observa en los estudiantes un claro compromiso social.

A pesar de que en varios estudiantes prima un paradigma tradicional (médico-clínico) acerca de la discapacidad, existe un enfoque más renovado en conceptos como necesidades educativas especiales, adecuaciones curriculares e inclusión. Sin embargo, reclaman la falta de práctica e implementación de ellos en situaciones educativas concretas en la escuela.

Cabe resaltar la valoración positiva que hacen en general de su formación, que a pesar de no desarrollar competencias específicas en el área, algunos declaran poseer otras competencias que les permitirían adaptarse a situaciones nuevas y resolver problemas, entre ellos, dar una respuesta adecuada a los estudiantes integrados en sus aulas. Lo anterior no deja de lado la responsabilidad educativa de formar profesionales integrales, capaces de construir con la diversidad del alumnado aprendizajes de calidad. Dicha investigación refleja la necesidad de implementar planes y programas de estudio que desarrollen competencias profesionales en los futuros docentes para hacer del aula un espacio de real inclusión.

En la medida que la educación sea cada vez más abierta a la diversidad, se irá avanzando en una sociedad menos discriminadora, donde la diferencia no constituya un obstáculo para la participación sino un valor de riqueza en las interacciones. En este sentido, caminar en procesos de integración escolar, constituye un paso en hacer de esta sociedad una más inclusiva y por ello más justa, donde los docentes tenemos un rol ineludible que cumplir.

\section{REFERENCIAS BIBLIOGRÁFICAS}

\section{LIBROS}

Arnais, P. (2003). Educación Inclusiva: Una escuela para todos. Málaga: Editorial Aljibe.

Bellei, C., Muñoz, G., Pérez, L.M. \& Raczynski, D. (2004). ¿Quién dijo que no se puede?, Escuelas efectivas en sectores de pobreza. UNICEF. Santiago, Chile.

Marchesi, A. (2001). La práctica de las escuelas inclusivas. En C. Coll, A. Marchesi, \& J. Palacios, Desarrollo Psicológico y Educación 3: Trastornos del desarrollo y necesidades educativas especiales (45-67) Madrid: Alianza Editorial.

Sola, T. (1997). La formación inicial y su incidencia en la educación especial. En Sánchez, A. \& Torres, J., Educación especial 1. Una perspectiva curricular, organizativa y profesional. Madrid: Editorial Pirámide.

Stainback, S. \& Stainback, W. (1999). Aulas Inclusivas. Madrid: Editorial Narcea.

\section{ARTÍCULOS REVISTAS}

Alvarez, M., Castro, P., Campo, M.A. y Martino, E. (2005). Actitudes de los maestros ante las necesidades educativas específicas. Revista Psicothema, Vol. 17, n. 04, 601-606.

Blanco, R. (1999). Hacia una escuela para todos y con todos. Boletín 48 Proyecto Principal de Educación, UNESCO. 
Burke, K. \& Sutherland, C. (2004). Attitudes toward inclusion: Knowledge vs. Experience. Education. Año 2, n. 125, 163-172.

García, J. y Alonso, J.C. (1985) Actitudes de los maestros hacia la integración escolar de niños con necesidades especiales. Revista Infancia y Aprendizaje, n. 30, 51-68.

Montañés, J., Jiménez, J., Blanc, P. \& González, M. (1990). Estudio sobre las actitudes ante la integración escolar en al Provincia de Albacete. Revista Ensayos: de la Escuela Universitaria de Magisterio de Albacete, n. 4, 137-154.

Tenorio S. (2009). Representaciones Sociales de la Integración Escolar: Miradas en Tensión. Revista Perspectivas Educacionales, nº 9, 209-207. Facultad de Filosofía y Educación UMCE, Santiago, Chile.

\section{DOCUMENTOS OFICIALES}

MINEDUC (2005). Política de Educación Especial. Santiago, Chile.

MINEDUC (2009). Decreto No 0170. Santiago, Chile.

\section{REVISTAS Y DOCUMENTOS ELECTRÓNICOS}

Brunner, J.J. \& Elacqua, G. (2004). Factores que inciden en una educación efectiva. Evidencia internacional. Revista Virtual La educación. Año XVLIII-XLIX, n. 139-140,I-II. Organización de Estados Americanos. OEA. (En línea). Consultado: (6, enero,2008)Disponible en:<http:// www.educoas.org/portal/bdigital/laeducacion/139/pdfs/139pdf1.pdf>

Educación 2020 (2009). Hoja de ruta 2009-2020. Se acabó el recreo. (En línea). Consultado (4, enero, 2011). Disponible en: http://www.educacion2020.cl/

MINEDUC (2010a). Panel de Expertos para una Educación de Calidad. Propuestas para fortalecer la profesión docente en el sistema escolar chileno. (En línea). Consultado: (7, octubre, 2010). Disponible en: www.mineduc.cl/biblio/.../201007091211380.Informe\%20final.

MINEDUC (2010b). Avances Proceso de Implementación de la Política Nacional de Educación Especial 2006-2010. Santiago, Chile. (En línea). Consultada: (29, Abril, 2010.). Disponible en: http://www.mineduc.cl/biblio/documento/201004121228150.Educacion\%20especial\%20 2006\%20-\%202009.pdf

MINEDUC-UMCE (2007). Calidad de la Integración Escolar y Factores Asociados. (En línea). Consultado: (22, Abril, 2010). Disponible en: http://200.68.0.250/usuarios/edu.especial/File/ UMCEFINAL.pdf

Murillo, F.J. (2003). Una panorámica de la investigación Iberoamericana sobre eficacia escolar. Revista Electrónica Iberoamericana sobre Calidad, Eficacia y Cambio en Educación, vol. 1, núm. 1. consultada el 5 de septiembre, 2010. En: www.inacap.cl/tportal/portales/.../6_ EfectividadEducativa.pdf

Ocde (2009). El DPD desde la mirada de los docentes, Boletín Especial, n. 51-52, GTD-PREAL. Versión electrónica, consultada el 29 de Abril de 2010. En: http://www.oei.es/noticias/spip. php?article6447

Tenorio, S. (2005). La Integración escolar en Chile: Perspectiva de los docentes sobre su implementación, Revista Electrónica Iberoamericana sobre Calidad, Eficacia y Cambio en Educación (REICE), vol. 3, n. 1(e), 823-83. Consultada el 25 de Abril, 2010. En: http://www.ice.deusto. es/RINACE/reice/Vol3n1_e/Tenorio.pdf

\section{MEMORIA DE TÍTULO Y TESIS}

Gonzalez G., Herrera J. \& Jara E. (2011). El abordaje de temáticas de integración escolar y estrategias didácticas para el trabajo de alumnos con necesidades educativas especiales, en la 
formación de estudiantes de distintas carreras de la UMCE, Memoria de título no publicada, Universidad Metropolitana de Ciencias de la Educación, Santiago de Chile.

Tenorio, S. (2007). Las Representaciones Sociales de Profesores Básicos de las Comunas De Ñuñoa y Macul Acerca de la Integración Escolar. Tesis Doctoral no publicada, Pontificia Universidad Católica de Chile, Santiago. 
